\title{
THE ANNULAR GAP: GAMMA-RAY \& RADIO EMISSION OF PULSARS
}

\author{
G. J. QIAO \\ Department of Astronomy, Peking University \\ Beijing, 100871, China \\ gjn@pku.edu.cn \\ Y. J. DU \\ National Astronomical Observatories \\ Chinese Academy of Sciences, Jia 20 Datun Road \\ Beijing 100012, China \\ dyj@nao.cas.cn \\ J. L. HAN \\ National Astronomical Observatories \\ Chinese Academy of Sciences, Jia 20 Datun Road \\ Beijing 100012, China \\ hjl@nao.cas.cn \\ R. X. XU \\ Department of Astronomy, Peking University \\ Beijing, 100871, China \\ r.x.xu@pku.edu.cn
}

\begin{abstract}
Pulsars have been found more than 40 years. Observations from radio to gamma-rays present abundant information. However, the radiation mechanism is still an open question. It is found that the annular gap could be formed in the magnetosphere of pulsars (neutron stars or quark stars), which combines the advantages of the polar cap, slot gap and outer gap models. It is emphasized that observations of some radio pulsars, normal and millisecond gamma-ray pulsars (MSGPs) show that the annular gap would play a very important role. Here we show some observational and theoretical evidences about the annular gap. For example, bi-drifting sub-pulses; radio and gamma-ray millisecond pulsars and so on.
\end{abstract}

Keywords: Neutron star; quark star magnetosphere; non-thermal radiation mechanisms.

\section{Introduction: The Annular Gap}

According to the traditional models, the structure and acceleration region of a pulsar magnetosphere are related to pulsars' nature, i.e. neutron stars (NSs) or quark star (QSs). But regardless of NSs or QSs, the annular gap will play an important role. The annular region is defined by the critical field lines that cross the null charge surface (NCS) and the last open field lines (see Fig. 1). 
High binding at the surface of NSs. If the binding energy per ion in the NS surface is as large as $10 \mathrm{keV}$, then ions will not be released. For an anti-parallel rotator, that is $\boldsymbol{\Omega} \cdot \mu<0$, a polar vacuum core gap can be formed (Rurderman \& Sutherland 1975, hereafter RS75), where $\boldsymbol{\Omega}$ is the angular velocity and $\mu$ is the magnetic dipole moment of a pulsar. For the fully charge-separated magnetospheres, the charges with only one-sign can exist at a given location, the negative charged particles can not pass the annular regions with positive GJ charge density (Goldreich \& Julian 1969). In this case, the outer gap will form around the null charge surface (Holloway 1973; Krause-Polstorff \& Michel 1985; Cheng et al. 1986).

Low binding at the surface of NSs. More accurate variational calculations (e.g. Flowers et al. 1977; Kössl et al. 1988) have revised the binding energy downward to only $\mathrm{keV}$. In this case, both the negative and positive charged particles will flow out freely from the neuron star surface. The space-charge-limited flow (SCLF) can be taking place (Sturrock 1971; Arons \& Scharlemann 1979; Harding, 1981). It is noted that the assumption of a fully charge separated magnetosphere is also used in the SCLF model.

Quark star. If pulsars are bare strange quark stars, the inner core and annular gaps can form for both cases of $\boldsymbol{\Omega} \cdot \mu<0$ and $\boldsymbol{\Omega} \cdot \mu>0$ (Xu et al. 1999; Xu et al. 2001; Qiao et al. 2004a).

In this paper we will show some observational evidences related to the annular gap.

\section{The Annular Gap: Normal Pulsars and MSGPs}

The open field line region of pulsar magnetosphere can be divided into two parts by the critical field lines (see Fig.1). The polar cap radius is $r_{p}=\left(\frac{2 \pi R^{3}}{p c}\right)^{1 / 2}$, which is corresponding to the outer rim of the annular gap at the surface of the neutron stars. Here $R$ is the radius of the neutron star, $p$ and $c$ are the pulsar period and the light speed, respectively. The radius of inner rim of the the annular gap at the surface is $r_{i n} \sim 0.74 r_{p}$. So the width of the annular gap is $\Delta r \sim 0.26\left(\frac{2 \pi R^{3}}{p c}\right)^{1 / 2}$. One can see that the annular gap will be wider for short-period pulsars. Calculations show that in the annular region the particle acceleration potential is much higher than that in the core region (Qiao et al. 2007).

Due to the high sensitivity and large effective collecting area, nearly 100 new $\gamma$-ray pulsars have been discovered by the Large Area Telescope (LAT) of Fermi, including $\gamma$-ray-only pulsars and a new population of millisecond pulsars (MSPs) (e.g. Abdo et al. 2010). With these excellent data, we have an opportunity to well study the high energy emission regions, which shows that the annular region plays a very important role (Du et al. 2010; 2011; 2012). The main results are as follows:

1. We have explored the formation reason of acceleration electric field $\left(E_{\|}\right)$in the annular gap. As Du et al. (2011) noted that, the charged particles can not co-rotate with a neutron star near the light cylinder, and must flow out from the magnetosphere. To keep the whole system charge-free, the neutron star surface must have the charged particles flowing into the magnetosphere. We found that the $E_{\|}$ 


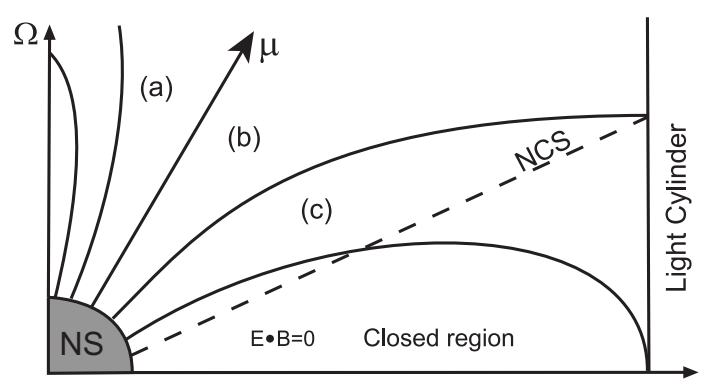

Fig. 1. A diagram for the annular gap and the core gap for particle acceleration. CFL stands for the critical field line across the intersection of the null charge surface and the light cylinder, NCS for the null charge surface, and LOF for the last open field line. (b) is the core region; (c) is the annular region, see Qiao et al. (2007).

is strongly correlated with the local magnetic field $B_{\mathrm{LC}}$ at an altitude of $R_{\mathrm{LC}}$. It has been found that the Fermi $\gamma$-ray pulsars can be young pulsars and millisecond pulsars which have a high $B_{\mathrm{LC}}$. This means that the acceleration electric field $E_{\|}$ in a pulsar magnetosphere is related to the observed Fermi $\gamma$-ray emission from pulsars.

2. The multi-wavelength pulsed emission from the Crab and Vela pulsar can be well explained in the annular gap and core gap model. Fermi observations reveal that the Vela pulsar has two sharp peaks (P1 and P2) in the light curve with a phase separation of 0.42 and a third peak (P3) in the bridge. The location and intensity of P3 are energy-dependent. These detailed features can be precisely reproduced by our annular gap model (Du et al. 2011). Furthermore, the famous Crab pulsar with phase-aligned multi-wavelength light curves can also be well explained by the annular gap model (Du et al. 2012). The modeled radio and $\gamma$-ray light curves for PSR J0437-4715 in the two-pole annular gap model are shown in Fig.2. Both radio and $\gamma$-ray emission is generated in the annular gap region, the region for the radio emission is mainly located at a higher altitude than the one of $\gamma$-ray emission.

3 . For the fully charge-separated magnetospheres, it is conventionally assumed that the outer gap will be formed around the null charge surface. This brings about two questions: 1) When the outer gap is initially forming, should the influence of particles flowing downwards to the NS surface be taken into account? 2) When the pairs are produced in the outer gap, would the "fully charge-separated magnetospheres" be destroyed by the pairs? Then a neutral magnetosphere will be formed with a dynamical equilibrium process. From the both cases, one can see that the outer gap can be hardly formed.

\section{Pulsar Radio Observations and the Annular Gap}

Some observational facts in radio bands shown that the existence of the annular gap, here we show two of them: Bi-drifting Sub-pulse Phenomenon and wide emission beams of Gamma-ray pulsars. 


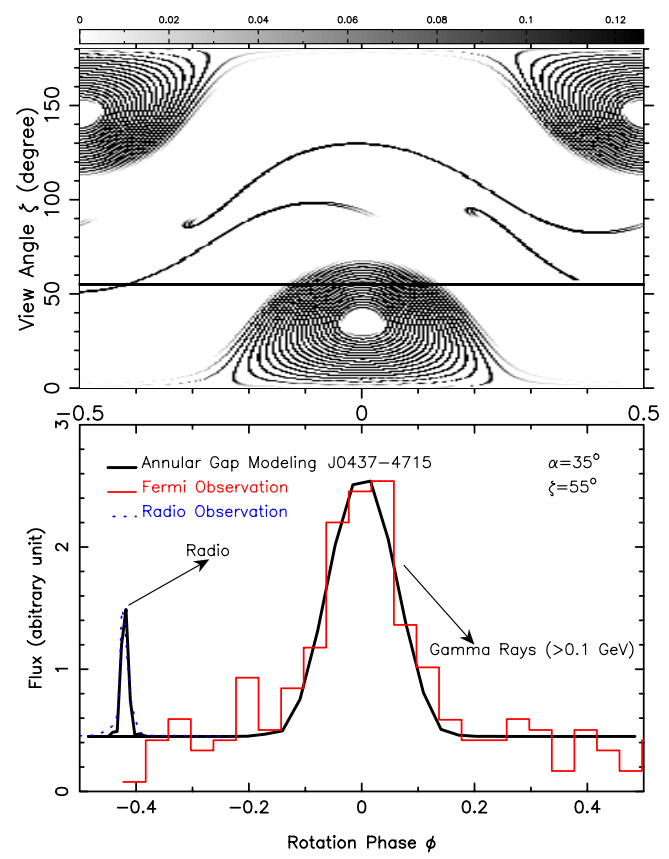

Fig. 2. The jointly modeled radio and $\gamma$-ray light curves (black solid lines) for the millisecond pulsar PSR J0437-4715. The radio emission is produced from a higher and narrower region in the annular gap region of the same magnetic pole as the $\gamma$-ray.

\section{The Bi-drifting Sub-pulse Phenomenon}

Observational evidences show that the radio emission of some pulsars with systematically drifting sub-pulses or periodic intensity modulations (Deshpande \& Rankin 1999, 2001; Vivekanand \& Joshi 1999). The drifting sub-pulse phenomena can be understood based on the drifting sparking in the inner polar vacuum gap (RS75; Gil \& Sendyk 2000; Qiao et al. 2004b). Besides the drifting phenomena, the observed radio emission beams (that are core and conal beams and so on, see Rankin 1983,1993; Lyne \& Machester 1988), linear and circular polarizations, the pulse profiles changing with frequencies can be understood through the inner gap sparking, it is called as the Inverse Compton Scattering (ICS) model(Qiao \& Lin 1998; Xu et al. 2000; Qiao et al. 2001), but the observed "Bi-drifting" sub-pulse phenomenon (the sub-pulses are moving in two opposite dedication ) is a challenge of pulsar radiation theories.

In the classical subpulse-drifting model, sub-pulse patterns are manifestations of some sparks passing along a ring (carousel) in the polar cap region circulating around the magnetic axis (RS75). A new drifting pulsar, PSR J0815+09, was discovered in the Arecibo drift-scan searches (McLaughlin et al. 2004), which is so-called "bi-drifting" phenomenon. This phenomenon poses a great challenge to the RS75 model and its relevant models, since in all these models, the drifting direction in 
spark ring is expected to be the same. Qiao et al. (2004b) pointed out that the bidrifting phenomenon is a newly observed touchstone to test the radiation theories and the surface physical properties of pulsars. Their simulated results supported the coexistence of the annular gap and the core gap.

\section{Wide Radio Emission beam for Gamma-ray pulsars}

Ravi, Manchester \& Hobbs (2010) investigate the radio and $\gamma$-ray beaming properties of normal and millisecond pulsars. They found that for high $\dot{E}$ pulsars, the radio emission is originated in wide beams from the vicinity of the null-charge surface. The null charge surface in just in the region of the annular gap region, which is consistent with our annular gap model, and it is a existence evidence of the annular gap.

\section{Conclusion and Discussion}

There is no prior justifications for a fully charge-separated magnetosphere. Even if the magnetosphere is initially fully charge-separated, the pair plasma generated from the acceleration region will soon fill regions in the annular flux tube, leading to a quasi-neutral plasma. In this case the traditional outer gap can not be formed. The annular gap is very important for the normal and MSGPs (Du et al. 2010, 2011, 2012).

If pulsars are QSs, the inner core and annular gaps can be formed regardless of $\boldsymbol{\Omega} \cdot \mu<0$ or $\boldsymbol{\Omega} \cdot \mu>0$. In this case, observed Bi-drifting can be understand naturally. If pulsars are neutron stars, free flow cab be formed, pair sparking can taking place in core and annular region. But no paper shows that the pulse drifting can take place or not in this case.

\section{Acknowledgments}

We are very grateful to Dr. K. J. Lee for his valuable contribution. This work is supported by NSF of China (10821061, 10573002, 10778611, 10773016, 11073030 and 10833003) and the Key Grant Project of Chinese Ministry of Education (305001). This work is partly supported by China Ministry of Science and Technology under State Key Development Program for Basic Research (2012CB821800).

\section{References}

Abdo, A. A., et al. 2010, ApJS, 187, 460.

Arons, J. 1981, ApJ, 248, 1099.

Arons, J. \& Scharlemann,E.T., 1979, ApJ, 231, 854.

Cheng, A. F. \& Ruderman, M., 1980, ApJ, 235, 576.

Cheng, K. S., Ho, C. \& Ruderman, M., 1986, ApJ, 300, 500.

Deshpande, A. A. \& Rankin, J. M., 1999, ApJ, 524, 1008.

Deshpande, A. A. \& Rankin, J. M., 2001, MNRAS, 322, 438.

Du, Y. J., Qiao, G. J., Han, J. L., Lee, K. J. \& Xu, R. X., 2010, MNRAS, 406, 2671.

Du, Y. J., Han, J. L., Qiao, G. J. \& Chou, C. K., 2011, ApJ, 731, 2. 
Du, Y. J., Qiao, G. J. \& Wang W., 2012, ApJ, in press.

Fawley, W. M., Arons, J. \& Scharlemann, E. T., 1977, ApJ, 217, 227.

Gil, J. A. \& Sendyk, M., 2000, ApJ, 541, 351.

Gil, J. A. \& Mitra, D., 2001, ApJ, 550, 383.

Gil, J. A. \& Melikidze, G. I., 2002, ApJ, 577, 909.

Goldreich, P. \& Julian, W. H., 1969, ApJ, 157, 869.

Harding, A. K., 1981, ApJ, 245, 267.

McLaughlin, M. A., Lorimer, D. R., Champion, D. J., et al. 2004, in Young Neutron Stars and Their Environments IAU Symposium, Vol. 218 (eds. F. Camilo and B. M. Gaensler) (astro-ph/0310454).

Muslimov,A.G. \& Harding, A. K., 2003, ApJ, 588, 430.

Holloway, N. J., 1973, Nature, 246, 6.

Kössl, D., Wolff, R.G., Miller, E. \& Hillebrandt, W., 1988, A\&A, 205, 347.

Krause-Polstorff, J. \& Michel, F.C., 1985, A\&A, 144, 72.

Levinson, A., Melrose, D., Judge, A. \& Luo, Q. H., 2005, ApJ, 631, 456.

Lyne, A. G., \& Machester, R. N., 1988, MNRAS, 234, 477.

Michel, F. C., 1974, ApJ, 192, 713.

Qiao, G. J. \& Lin, W. P., 1998, A\&A, 333, 172.

Qiao, G. J., Liu, J.F., Zhang, B. \& Han, J. L., 2001, A\&A, 377, 964.

Qiao, G. J., Lee, K. J., Wang, H. G., Xu, R. X. \& Han, J. L., 2004a, ApJ, 606, L49.

Qiao, G. J., Lee, K. J., Zhang, B., Xu, R. X. \& Wang, H. G., 2004b, ApJ, 616, L127.

Qiao, G. J., Lee, K. J., Zhang, B., Wang, H. G., Xu, R. X., 2007, CjAA, 7, 496.

Rankin, J. M., 1983, ApJ, 274, 333.

Rankin, J. M., 1993, ApJ, 405, 285.

Ravi, V., Manchester R. N. \& Hobbs, G., 2010, ApJ, 716, L85.

Ruderman, M. A., \& Sutherland, P. G., 1975, ApJ, 196, 51.

Scharlemann, E. T., Arons, J. \& Fawley, W. M., 1978, ApJ, 222, 297.

Sturrock, P. A. 1971, ApJ, 164, 529.

Vivekanand, M. \& Joshi, B. C., 1999, ApJ, 515, 398.

Wang, H. G., Qiao, G. J., Xu, R. X. \& Liu, Y., 2006, MNRAS, 366, 945.

Xu, R. X., Liu, J. F. \& Han, J.L. \& Qiao, G. J., 2000, ApJ, 535, 354.

Xu, R. X., Qiao, G. J. \& Zhang, B., 1999, ApJ, 522, L109.

Xu, R. X., Zhang, B. \& Qiao, G. J., 2001, Astroparticle Physics, 15, 101. 